\title{
Simulations of eddy kinetic energy transport in barotropic turbulence
}

\author{
Ian Grooms* \\ Department of Applied Mathematics, University of Colorado, Boulder
}

(Dated: October 17, 2017)

\begin{abstract}
Eddy energy transport in rotating, two-dimensional turbulence is investigated using numerical simulation. Stochastic forcing is used to generate an inhomogeneous field of turbulence, and the time-mean energy profile is diagnosed. An advective-diffusive model for the transport is fit to the simulation data by requiring the model to accurately predict the observed time-mean energy distribution. Isotropic harmonic diffusion of energy is found to be an accurate model in the case of uniform, solid-body background rotation (the $f$-plane), with a diffusivity that scales reasonably well with a mixing-length law $\kappa \propto V \ell$ where $V$ and $\ell$ are 'characteristic' eddy velocity and length scales. Passive tracer dynamics are added, and it is found that the energy diffusivity is $75 \%$ of the tracer diffusivity. The addition of a differential background rotation with constant vorticity gradient $\beta$ leads to significant changes to the energy transport. The eddies generate and interact with a mean flow that advects the eddy energy. Mean advection plus anisotropic diffusion (with reduced diffusivity in the direction of the background vorticity gradient) is moderately accurate for flows with scale separation between the eddies and mean flow, but anisotropic diffusion becomes a much less accurate model of the transport when scale separation breaks down. Finally, it is observed that the time-mean eddy energy does not look like the actual eddy energy distribution at any instant of time. In the future, stochastic models of the eddy energy transport may prove more useful than models of the mean transport for predicting realistic eddy energy distributions.
\end{abstract}

\section{INTRODUCTION}

Mesoscale eddies - long-lived vortices with diameters of $150 \mathrm{~km}$ or so - are ubiquitous in the world oceans, dominating the eddy kinetic energy [1]. Global ocean models are available with horizontal grid scale sufficient to begin to resolve these eddies [2], but their computational expense is prohibitive for ensemble simulations and long-running simulations. Ocean general circulation models with horizontal grid scales on the order of $100 \mathrm{~km}$, which do not resolve mesoscale eddies, continue to be used for a variety of purposes like the CESM Large Ensemble [3], the CORE model intercomparisons [4], and climate-model data assimilation [5]. Mesoscale eddies have a significant impact on the distribution of active and passive tracers in the global ocean, and their effects must be parameterized in models that do not resolve them. The most common and successful framework for parameterizing the tracer transport affected by mesoscale eddies is the Gent-McWilliams (GM) parameterization [6, 7], which is typically used in conjunction with an isopycnal mixing parameterization proposed by Martha Redi [8]. The GM parameterization models the adiabatic rearrangement of tracers by mesoscale eddies using an eddy-induced bolus velocity [7] whose amplitude is proportional to a diffusivity $\kappa_{\mathrm{GM}}$. The Redi parameterization models the mixing of tracers along surfaces of constant density, and the Redi diffusivity is often set equal to $\kappa_{\mathrm{GM}}$.

A great deal of effort has been expended developing models for the spatial and temporal variation of the GM coefficient $\kappa_{\mathrm{GM}}$. Common parameterizations of $\kappa_{\mathrm{GM}}$ set $\kappa_{\mathrm{GM}} \propto \ell^{2} / T$ or $\kappa_{\mathrm{GM}} \propto V \ell$ where $V$, $\ell$, and $T$ are velocity, length, and time scales of the eddies that are given as functions of the resolved large scale flow $[9,10]$. For example, the length scale $\ell$ can be approximated by the local deformation radius, following Stone [11]. The characteristic length, time, and velocity scales of mesoscale eddies vary considerably over the global ocean [1, 12-14], and any successful model for $\kappa_{\mathrm{GM}}$ should reflect this. In particular, parameterizations of the form $\kappa_{\mathrm{GM}} \propto V^{2} T$, where $V^{2}$ is proportional to the eddy energy, have been highlighted as particularly effective in several recent studies including [15-23].

Parameterizations that specify the subgrid scale fluxes and stresses solely in terms of properties of the large scale flow are, in the terminology of turbulence modeling [24], 'zero-equation' models. By contrast, 'one-equation' models evolve some statistic (typically energy) of the subgrid scales along with the large scale flow variables, and it is possible to build models that keep track of more than one subgrid scale statistic (e.g. K- $\epsilon$ models [24] and the level 2.5 and higher Mellor-Yamada models [25]). Of the above-cited studies highlighting the importance of eddy energy in mesoscale parameterization, only two have proposed one-equation models for the eddy energy: Eden and Greatbatch [16] in a complete ocean model, and Marshall and Adcroft [17] in a highly idealized model. The key assumption of such models is that transport of subgrid scale energy plays an important role in setting the global distribution of

*ian.grooms@colorado.edu; www.colorado.edu/amath/grooms 
30 subgrid scale energy. (In contrast, Cessi [15] and Jansen et al. [18] develop zero-equation parameterizations based on the assumption that the subgrid-scale energy is set by a balance between local generation and dissipation.) Grooms et al. [26] and Chen et al. [27] recently found evidence of nonlocality in the mesoscale eddy energy budget of idealized model simulations and in a global ocean state estimate, respectively. A multiple-scale asymptotic model also predicted the importance of nonlocal transport of eddy energy [28]. These three studies [26-28] suggest that successful prediction

35 of the global structure of mesoscale energy will require (at least) a one-equation model that takes energy transport into consideration.

In addition to these three direct studies of mesoscale eddy energy nonlocality, several studies have investigated the propagation of coherent mesoscale eddies in (e.g. [1, 29-33], to cite just a few). These studies strongly suggest that eddy energy transport in idealized models and the real ocean has a distinctive anisotropic character, but none of them attempts to relate coherent eddy propagation directly to eddy energy transport. Perhaps more importantly, the studies focus on coherent eddies, which form only a part of the mesoscale 'eddy' field, which includes both coherent and incoherent motions. This conceptual difference has recently been highlighted in [34], where coherent eddies were found to contribute less than $1 \%$ to the total mesoscale meridional tracer transport in the East Pacific.

Subgrid-scale energy transport has been studied and modeled in many fluid systems. The simplest model for the incompressible three-dimensional turbulence go back at least to Lilly [35], and are employed by both Eden and Greatbatch [16] and Marshall and Adcroft [17]. In the context of incompressible three-dimensional turbulence, this type of transport model can be motivated theoretically under the assumption of asymptotic scale separation between the subgrid scale eddies and the resolved flow [36-38], but the relevance of these analyses to mesoscale eddy dynamics $5_{0}$ is unclear. While it would be very convenient to model subgrid scale energy transport as the sum of large-scale advection and diffusion, there is almost no experimental or theoretical support for this model in the case of mesoscale eddy dynamics.

This question has been addressed by the author in a previous study [39] using numerical experiments in the simplest possible setting: rotating, two-dimensional incompressible turbulence on an $f$-plane. In contrast to threedimensional incompressible turbulence, where vortex stretching cascades energy to smaller scales, in two-dimensional incompressible turbulence the energy is transferred towards larger scales where it condenses into coherent eddies, while the enstrophy (conserved in two dimensions, but not three) is cascaded towards smaller scales and dissipation. The inverse cascade of energy is halted only by the domain size or by frictional effects acting directly at the eddy scales; in an oceanic context this friction is afforded through interaction with the viscous bottom boundary layer.

The simulations in [39] were performed in a periodic domain, with a region of localized stochastic forcing in the center. They do not generate a time-mean flow, and the entire field of dynamics generated by the stochastic forcing is considered to be part of the eddy field, i.e. it would be subgrid-scale in an ocean model with a grid scale on the order of $100 \mathrm{~km}$. The length scale of the eddies is smaller than the region of stochastic forcing, and the time-mean eddy energy thus varies on spatial scales larger than the eddies. The time-mean eddy energy profile was diagnosed

was found that modeling the eddy energy transport as isotropic harmonic diffusion led to accurate predictions of the mean eddy energy. Furthermore, the energy diffusivity $\kappa$ was reasonably well predicted by a mixing-length approximation $\kappa \propto V \ell$, where $V$ is a root-mean-square (RMS) velocity and $\ell$ is a characteristic eddy length scale.

The current investigation extends the previous one in three ways. First, simulations are performed at higher energies and with a greater scale separation between the scale of the eddies and the scale of the region of localized forcing; the results confirm those reported earlier. Second, a passive tracer is added to the simulations for the purpose of comparing the rate of energy diffusion to the rate of passive scalar diffusion; it is found that the energy diffusivity is approximately $75 \%$ of the tracer diffusivity across a range of simulations. Finally, a 'planetary' background vorticity gradient $\beta$ is added to the simulations. This final addition significantly alters the energy transport to the extent that even an anisotropic diffusion model is only moderately accurate.

The experimental configurations, numerical methods, and analytical framework are described in section II. Results of the simulations are presented, and models for the divergence of the energy flux are tested in section III, and conclusions are offered in section IV.

\section{METHODS}

\section{A. Continuum dynamics}

The model adopted here is barotropic vorticity dynamics on a $\beta$-plane

$$
\partial_{t} q+\boldsymbol{u} \cdot \nabla q+\beta v=F_{q}-q+\nu \nabla^{2} q
$$


where $q=\nabla^{2} \psi$ is the vorticity, $\psi$ is the streamfunction, $u=-\partial_{y} \psi$ and $v=\partial_{x} \psi$ are the $x$ and $y$ components of velocity, $\nu$ is the nondimensionalized turbulent viscosity coefficient, and $F_{q}$ is a spatially-correlated Gaussian stochastic forcing term that is white in time and uncorrelated with the flow. In a periodic domain this model is distinguished from two-dimensional incompressible Navier-Stokes by the presence of linear friction ( $-q$ in Eq. 1), which is a common model of the frictional effect of the viscous Ekman layer in a rotating fluid, and by $\beta$, which is the gradient of a background 'planetary' vorticity. The equation is nondimensional with time scale equal to the rate of linear friction and length scale chosen so that the square, doubly-periodic domain has width $L$. Simulations in [39] used either $L=2$ or $L=3$; all new simulations reported here use $L=2$. This length scale is only meaningful in comparison with the length scale of the stochastic forcing, discussed below; in particular, it needs to be large enough to accommodate a scale separation between the eddy length scale and the scale over which the eddy energy varies. The use of a Laplacian viscosity as opposed to a higher-order viscosity or a spectral filter is motivated by a desire to accurately compute the viscous energy dissipation rate, which can be difficult for more common small-scale closures.

The stochastic forcing does not model any physical process; its presence is solely for the purpose of providing an energy source. The energy of real ocean eddies typically comes from interaction with a larger-scale flow, with the can be precisely controlled. It is hoped that the eddy energy transport will be 'generic' or 'universal' in the sense of being largely insensitive to the details of the energy generation, so that the behavior observed here can be cautiously extrapolated to more complicated and more realistic scenarios.

The stochastic forcing is spatially localized, so that energy transport can be observed from the more-energetic forcing region to the less-energetic unforced region. In general, a localized vorticity forcing will not necessarily be equivalent to localized momentum force though, and care must be taken to ensure that the energy forcing implied by the vorticity forcing is spatially localized. The stochastic vorticity forcing $F_{q}$ is therefore constructed as the Laplacian of a streamfunction forcing, $F_{q}=F_{0} \nabla^{2} F_{\psi}$, where $F_{\psi}$ is zero outside a circle with diameter 1 at the center of the domain and $F_{0}$ controls the amplitude of the forcing; as a result, both the vorticity forcing and the implied ergy forcing are zero outside the forcing circle. (The use of a stochastic forcing that is white in time allows the energy forcing implied by $F_{q}$ to be determined a priori.) The streamfunction forcing is constructed in two steps: first a zero-mean homogeneous isotropic Gaussian random field is generated, of the kind commonly used to force homogeneous turbulence, and second this field is multiplied by a smooth window function that tapers it to zero outside the forcing circle. Specifically $F_{\psi}=M(r) \tilde{F}_{\psi}$, where $M(r)=\exp \left\{-4 r^{2} /\left(1-4 r^{2}\right)\right\}$ for $r<1 / 2$ and $M(r)=0$ for $r \geq 1 / 2$, and where $\tilde{F}_{\psi}$ is the homogeneous isotropic random field. In the previous study [39] the spectrum of $\tilde{F}_{\psi}$ was proportional to $k^{-1} \exp \left\{-\left(k-k_{f}\right)^{2} / 2\right\}$ so that the kinetic energy forcing spectrum was peaked at wavenumber $k_{f}$. Previously-reported results ([39]) used peak forcing wavenumbers corresponding to wavelengths $\lambda_{f}=1 / 2,1 / 4$, $1 / 6$, and $1 / 8$. New simulations reported here use a spectrum that is proportional to $k^{-1} \mathrm{I}(k)$ where $I$ is the indicator function on a circular shell of width $2 \pi$ and centered on a particular wavenumber $k_{f}$, where the forcing wavelength is either $\lambda_{f}=1 / 8$ or $1 / 10$. The change in spectrum was made so that fewer random Fourier coefficients need to be sampled in order to construct the spatial random field. The field $\tilde{F}_{\psi}$ is normalized so that the energy forcing has amplitude $F_{0}^{2}$ at the center of the domain.

To investigate passive tracer transport in these inhomogeneous flows, a tracer equation was added to the dynamics

$$
\partial_{t} C+\boldsymbol{u} \cdot \nabla(C+x)=\nu \nabla^{2} C .
$$

The total tracer field is $C+x$, and tracer fluctuations $C$ are generated entirely by interaction of the velocity with the large-scale background tracer field. The amplitude of the gradient has been set to 1; gradients with different (nonzero) amplitudes can be rescaled to unity by rescaling $C$.

The tracer simulations used a different stochastic vorticity forcing $F_{q}$. The only difference in the forcing is that a different function is used to spatially-localize the forcing. The motivation for this change is that it is convenient to generate a turbulence field that is homogeneous in the same direction as the tracer transport. Thus, the streamfunction forcing is constructed as $F_{\psi}=m(y) \tilde{F}_{\psi}$, where

$$
m(y)=\left\{\begin{array}{cl}
1-e^{\frac{4}{40(y+1)^{2}-10}} & \text { for } y \leq-\frac{1}{2} \\
1 & \text { for }-\frac{1}{2} \leq y \leq \frac{1}{2} \\
1-e^{\frac{4}{40(y-1)^{2}-10}} & \text { for } y \geq \frac{1}{2}
\end{array}\right.
$$

\section{B. Energy transport}

This section presents a theoretical energetic analysis of the dynamical models described in the preceding section. The analysis proceeds under the assumption that the dynamics are ergodic in the sense that the long-time average 
is deterministic, i.e. that it does not depend on the particular realization of stochastic forcing, or on the initial condition. Under this assumption the mean kinetic energy equation can be obtained from Eq. 1 by multiplying by $-\psi$ and averaging. Repeated use of the chain rule is necessary $(f \nabla \cdot \boldsymbol{g}=\nabla \cdot(f \boldsymbol{g})-\boldsymbol{g} \cdot \nabla f$ where $f$ is a scalar function and $\boldsymbol{g}$ is a vector function). Since the stochastic forcing is white in time, the mean rate of energy input is independent of the flow. The mean energy equation takes the form

$$
\nabla \cdot \overline{\boldsymbol{F}}+2 \bar{E}=\bar{F}_{E}-2 \nu \bar{Z}
$$

where $\bar{E}=\frac{1}{2} \overline{|\boldsymbol{u}|^{2}}$ is the energy, $\bar{Z}=\frac{1}{2} \overline{q^{2}}$ is the mean enstrophy, $\overline{\boldsymbol{F}}$ is the mean energy flux, and $\bar{F}_{E}$ is the mean energy forcing rate. (Throughout the paper the term 'energy' is used to refer to $|\boldsymbol{u}|^{2} / 2$, despite the fact that the energy density is $\rho_{0}|\boldsymbol{u}|^{2} / 2$ and the total energy is the integral of the energy density over the domain.)

In [39] there was no mean flow, so the energy $\bar{E}$ was composed entirely of eddy energy. A time-mean velocity $\overline{\boldsymbol{u}}$ does develop in the simulations here with $\beta \neq 0$, and since the investigation is focused on eddy energy transport this needs to be taken into account. The mean eddy energy is thus defined as $\bar{e}=\overline{\left|\boldsymbol{u}^{\prime}\right|^{2}} / 2$ where $\boldsymbol{u}^{\prime}=\boldsymbol{u}-\overline{\boldsymbol{u}}$ is the eddy velocity and $\overline{\boldsymbol{u}}$ is the time-mean velocity. The eddy vorticity equation is obtained by subtracting the time-mean from Eq. 1, yielding

$$
\partial_{t} q^{\prime}+\boldsymbol{u}^{\prime} \cdot \nabla q^{\prime}-\overline{\boldsymbol{u}^{\prime} \cdot \nabla q^{\prime}}+\overline{\boldsymbol{u}} \cdot \nabla q^{\prime}+\boldsymbol{u}^{\prime} \cdot \nabla \bar{q}+\beta v^{\prime}=F_{q}-q^{\prime}+\nu \nabla^{2} q^{\prime} .
$$

An eddy energy equation can be obtained as above, yielding

$$
\nabla \cdot \overline{\boldsymbol{f}}+2 \bar{e}=\bar{F}_{E}-2 \nu \bar{z}+\overline{\mathcal{G}}
$$

where $\boldsymbol{f}$ is the mean eddy energy flux, $z=\overline{\left(q^{\prime}\right)^{2}} / 2$ is the eddy enstrophy, and $\overline{\mathcal{G}}$ is the rate of eddy energy generation through interaction with the mean flow. There are many ways to formulate analytical expressions for $\overline{\boldsymbol{f}}$ and $\overline{\mathcal{G}}$. For example, if one writes a particular formulation of $\overline{\boldsymbol{f}}$ and $\overline{\mathcal{G}}$, a new, different formulation can be obtained by adding $\overline{\boldsymbol{u}} \bar{e}$ to $\overline{\boldsymbol{f}}$ and adding $\nabla \cdot \overline{\boldsymbol{u}} \bar{e}$ to $\overline{\mathcal{G}}$. It is desirable to obtain a unique formulation that is distinguished by the fact that $\overline{\mathcal{G}}$ describes purely local generation of eddy energy and all nonlocal, transport effects are contained in $\nabla \cdot \overline{\boldsymbol{f}}$. The following derivation achieves such a unique formulation by investigating the mean energy equation, where the term $\overline{\mathcal{G}}$ also appears.

The velocity equations associated with Eq. 1 are

$$
\partial_{t} \boldsymbol{u}+\boldsymbol{u} \cdot \nabla \boldsymbol{u}+\left(f_{0}+\beta y\right) \boldsymbol{u}^{\perp}=-\frac{\nabla p}{\rho_{0}}+\boldsymbol{F}_{u}-\boldsymbol{u}+\nu \nabla^{2} \boldsymbol{u}
$$

where $\boldsymbol{u}^{\perp}=(-v, u), \rho_{0}$ is the constant fluid density, $\boldsymbol{F}_{u}$ is the stochastic velocity forcing, and the velocity is incompressible $\nabla \cdot \boldsymbol{u}=0$. (This equation does not make sense in the periodic domain because of the linear vorticity gradient, but this inconsistency is immaterial to the following discussion.)

The time-mean velocity equation is

$$
\overline{\boldsymbol{u}} \cdot \nabla \overline{\boldsymbol{u}}+\nabla \cdot\left(\overline{\boldsymbol{u}^{\prime} \otimes \boldsymbol{u}^{\prime}}\right)+\left(f_{0}+\beta y\right) \overline{\boldsymbol{u}}^{\perp}=-\frac{\nabla \bar{p}}{\rho_{0}}-\overline{\boldsymbol{u}}+\nu \nabla^{2} \overline{\boldsymbol{u}} .
$$

A time-mean flow can only be generated by a time-mean Reynolds stress $\overline{\boldsymbol{u}^{\prime} \otimes \boldsymbol{u}^{\prime}}$, which does not occur on the $f$-plane (i.e. when $\beta=0$ ). At first glance it would appear that the eddy velocity only appears in the time-mean equation through the Reynolds-stress divergence, but this is not precisely correct. The divergence of the Reynolds stress itself includes a divergent component that is balanced by an induced pressure gradient. Indeed, taking the divergence of Eq. 8 yields a Poisson equation for pressure

$$
\nabla^{2} \frac{\bar{p}}{\rho_{0}}=-\nabla \cdot\left[\overline{\boldsymbol{u}} \cdot \nabla \overline{\boldsymbol{u}}+\nabla \cdot\left(\overline{\boldsymbol{u}^{\prime} \otimes \boldsymbol{u}^{\prime}}\right)+\left(f_{0}+\beta y\right) \overline{\boldsymbol{u}}^{\perp}\right]
$$

Since the pressure equation is linear its solution can be decomposed into a sum of components associated with each of the terms on the right hand side of Eq. 9. Defining $\bar{p}_{u^{\prime}}$ to be a solution to

$$
\nabla^{2} \frac{\bar{p}_{u^{\prime}}}{\rho_{0}}=-\nabla \cdot\left[\nabla \cdot\left(\overline{\boldsymbol{u}^{\prime} \otimes \boldsymbol{u}^{\prime}}\right)\right]
$$

shows that the full eddy effect on the time-mean velocity is associated with both the Reynolds stress divergence and the associated pressure gradient. 
This becomes important when attempting to define the time-mean rate of energy exchange between the mean and eddies. The full eddy effect is arranged as a forcing term on the right hand side of the time-mean velocity equation of the form

$$
-\nabla \cdot\left(\overline{\boldsymbol{u}^{\prime} \otimes \boldsymbol{u}^{\prime}}\right)-\frac{\nabla \bar{p}_{u^{\prime}}}{\rho_{0}} .
$$

This eddy forcing term does not directly involve the mean velocity, and can be considered as a body force acting locally on the mean flow. (Briefly, note that the term isotropic as applied here to the eddy velocity field means $\overline{\left(u^{\prime}\right)^{2}}=\overline{\left(v^{\prime}\right)^{2}}$ and $\overline{u^{\prime} v^{\prime}}=0$. An isotropic eddy field generates a time-mean Reynolds stress equal to the eddy kinetic energy times a $2 \times 2$ identity matrix; it is straightforward to verify that the net eddy-induced body force (expression 11 ) is then zero, so an isotropic eddy field does not generate a mean flow.) The rate of energy injection by this body force is obtained by simply taking the dot product with $\overline{\boldsymbol{u}}$, yielding

$$
-\overline{\mathcal{G}}=-\overline{\boldsymbol{u}} \cdot\left[\nabla \cdot\left(\overline{\boldsymbol{u}^{\prime} \otimes \boldsymbol{u}^{\prime}}\right)+\frac{\nabla \bar{p}_{u^{\prime}}}{\rho_{0}}\right]
$$

where $\overline{\mathcal{G}}$ is the rate of eddy energy generation. This yields a purely local formula for $\overline{\mathcal{G}}$, which can be inserted into the time-mean local eddy energy equation, which becomes

$$
\nabla \cdot \overline{\boldsymbol{f}}+2 \bar{e}=\bar{F}_{E}-2 \nu \bar{z}+\overline{\boldsymbol{u}} \cdot\left[\nabla \cdot\left(\overline{\boldsymbol{u}^{\prime} \otimes \boldsymbol{u}^{\prime}}\right)+\frac{\nabla \bar{p}_{u^{\prime}}}{\rho_{0}}\right] .
$$

The reader may perhaps hope to next obtain a formula for the eddy energy flux $\overline{\boldsymbol{f}}$ that is associated with this particular formulation of the eddy/mean interaction term $\overline{\mathcal{G}}$. No such formula has been derived (though two are given in [39] for the case of zero mean flow). The ultimate goal of the current investigation is to predict the spatial ibution of eddy energy. Modeling the eddy energy flux (or its divergence) is only a means to that end. Rather than diagnose the mean eddy energy flux and compare modeled versus diagnosed fluxes, the approach here is to compare the mean eddy energy that would result from a particular flux model to the diagnosed mean eddy energy distribution. Rather than solve the 'forward' problem of finding a model of the flux that matches the observed flux, the approach here is to solve the 'inverse' problem of finding a model of the flux that implies an accurate time-mean eddy energy distribution.

Since eddy energy flux has been modeled in other contexts as the sum of mean advection and downgradient diffusion, the goal here is to test whether such a model is accurate in this context. The energy flux-divergence is thus modeled here as

$$
\nabla \cdot \overline{\boldsymbol{f}} \approx \overline{\boldsymbol{u}} \cdot \nabla \bar{e}-\nabla \cdot(\mathbf{K} \nabla \bar{e})
$$

where $\mathbf{K}$ is a $2 \times 2$ symmetric positive definite matrix. The simplest model is isotropic diffusion

$$
\mathbf{K}=\kappa \mathbf{I}
$$

where $\kappa$ is the eddy energy diffusivity. Symmetric, positive-definite $2 \times 2$ matrices can be written as a three-parameter family as follows

$$
\mathbf{K}=\left[\begin{array}{rr}
\cos (\theta) & -\sin (\theta) \\
\sin (\theta) & \cos (\theta)
\end{array}\right]\left[\begin{array}{cc}
\kappa_{1} & 0 \\
0 & \kappa_{2}
\end{array}\right]\left[\begin{array}{rr}
\cos (\theta) & \sin (\theta) \\
-\sin (\theta) & \cos (\theta)
\end{array}\right]
$$

This diffusion matrix describes diffusion along the direction $\theta$ counterclockwise from the $x$-axis at rate $\kappa_{1}$, plus diffusion along the orthogonal direction at rate $\kappa_{2}$. The eddy energy forcing rate $\bar{F}_{E}$ is known a priori, and the time-mean eddy enstrophy $\bar{z}$ and generation $\overline{\mathcal{G}}$ are diagnosed from simulations. Inserting the model Eq. 14 into Eq. 13 yields a linear equation for the time-mean eddy energy distribtion

$$
\overline{\boldsymbol{u}} \cdot \nabla \bar{e}_{\theta, \kappa}-\nabla \cdot\left(\mathbf{K} \nabla \bar{e}_{\theta, \kappa}\right)+2 \bar{e}_{\theta, \kappa}=\bar{F}_{E}-2 \nu \bar{z}+\overline{\boldsymbol{u}} \cdot\left[\nabla \cdot\left(\overline{\boldsymbol{u}^{\prime} \otimes \boldsymbol{u}^{\prime}}\right)+\frac{\nabla \bar{p}_{u^{\prime}}}{\rho_{0}}\right] .
$$

The solution $\bar{e}_{\theta, \kappa}$ is not equal to the observed time-mean eddy energy distribution $\bar{e}$, but the error can be minimized by optimizing over the parameters of the model, $\theta, \kappa_{1}$, and $\kappa_{2}$. The 'optimal' model of the eddy energy flux is obtained here by choosing parameters $\theta, \kappa_{1}$, and $\kappa_{2}$ to minimize the error between $\bar{e}_{\theta, \kappa}$ and $\bar{e}$. The error in the eddy energy distribution is optimized using the $L^{1}, L^{2}$, and $L^{\infty}$ norms; the optimal parameter values are typically very similar for the three norms, and results are presented for the $L^{2}$ norm unless noted otherwise.

In [39], there was no mean flow and the flux-divergence was modeled as an isotropic fractional diffusion $-\left(-\kappa \nabla^{2}\right)^{\alpha / 2} \bar{e}$. The optimal exponent $\alpha$ was invariably close to 2 , and there was little difference between the model prediction of $\bar{e}$ with $\alpha=2$ and with the optimal $\alpha \neq 2$. Since Laplacian diffusion is easier to implement than fractional diffusion as a parameterization in a fluid code, harmonic diffusion is the only model considered here. 
For the new simulations presented here the vorticity equation (Eq. 1) and tracer equation (Eq. 2) are solved using a dealiased Fourier spectral method with either $1536 \times 1536$ or $1280 \times 1280$ nonzero Fourier modes (i.e. these are the modes that remain after dealiasing), in a square domain of width $L=2$. The equations are integrated in time using the same fourth-order adaptive semi-implicit Runge-Kutta method described in [39]. In this method the Runge-Kutta scheme evolves only the deterministic dynamics; at the end of a Runge-Kutta step the forcing is added using an Euler approximation. This method is motivated by [40] where it was shown that one can achieve reasonable accuracy by applying a high-order method to the deterministic part (the 'drift' in the terminology of stochastic systems) and the Euler method to the white noise forcing whenever the noise is small compared to the deterministic part of the dynamics. This is fortunately the case in two-dimensional turbulence because of the inverse cascade and associated accumulation of energy. The nondimensional viscosity $\nu$ is chosen to depend on forcing amplitude and spatial discretization so that the energy dissipation by friction account for at least $90 \%$ of the total energy dissipation. The viscosity is sufficient to avoid accumulation of enstrophy at small scales, and ensures that the dynamics are, like large-scale ocean dynamics, largely insensitive to viscosity.

Three new experiments have been run with $\beta=0$ and without the tracer, extending the results of [39] to higher energies and smaller scales:

- Forcing scale $\lambda_{f}=1 / 8$, forcing amplitude $F_{0}=8$, viscosity $\nu=1.25 \times 10^{-4}, 1280 \times 1280$ Fourier modes

- $\lambda_{f}=1 / 8, F_{0}=10, \nu=1.3021 \times 10^{-4}, 1536 \times 1536$

- $\lambda_{f}=1 / 10, F_{0}=5, \nu=6.5104 \times 10^{-5}, 1536 \times 1536$

Six experiments have been run with $\beta=0$ and with the tracer:

- $\lambda_{f}=1 / 8, F_{0}=4.26, \nu=3.125 \times 10^{-5}, 1280 \times 1280$

- $\lambda_{f}=1 / 8, F_{0}=7.11, \nu=6.25 \times 10^{-5}, 1280 \times 1280$

- $\lambda_{f}=1 / 8, F_{0}=14.22, \nu=1.3021 \times 10^{-4}, 1536 \times 1536$

- $\lambda_{f}=1 / 10, F_{0}=3.91, \nu=2.278 \times 10^{-5}, 1536 \times 1536$

- $\lambda_{f}=1 / 10, F_{0}=7.84, \nu=4.556 \times 10^{-5}, 1536 \times 1536$

- $\lambda_{f}=1 / 10, F_{0}=15.66, \nu=6.5104 \times 10^{-5}, 1536 \times 1536$

Six experiments have been run with $\beta \neq 0$. Four simulations have been run with $\lambda_{f}=1 / 10, F_{0}=5$, $\nu=6.5104 \times 10^{-5}, 1536 \times 1536$ Fourier modes and with $\beta$ equal to 500,1000, 2000, and 3000. Two more have been run with $\lambda_{f}=1 / 8, F_{0}=10, \nu=1.3021 \times 10^{-4}, 1536 \times 1536$ Fourier modes and with $\beta$ equal to 1000 and 2000 .

The simulations are integrated until the time-mean energy and enstrophy distributions reach equilibria (the total energy and enstrophy equilibrate rapidly; only the spatial distribution converges slowly). This takes a long time; the low-energy regions are slowest to converge. Depending on the parameter values the simulations have to be run from 300 to 500 nondimensional time units; some simulations took nearly 10 million time steps. The longest simulations took several months of wall-clock time on 12 modern cores; the relatively small size of the spatial grid (in comparison with simulations of three-dimensional turbulence) means that there is minimal benefit, if any, from attempting to scale a single simulation to hundreds or thousands of processors. Instead, to speed convergence of the time averages, several simulations with the same parameter values can be run simultaneously, and their results are then averaged together. Symmetries of the problem have also been used to speed convergence. For example, in the simulations with tracers, the turbulence is homogeneous in the $x$-direction, so the long-time average has been augmented by an average over the $x$-direction (since the time average should in principle be independent of $x$ ). After these methods are applied to speed convergence, the computational cost is dominated by the need to resolve four disparate length scales in the simulation: the domain scale, the scale of the forcing region, the scale of the eddies, and the scale of viscous dissipation.

A numerical method is also required to compute solutions to Eq. 16. In cases where $\beta=0$ there is no mean flow, 250 and solutions can be directly calculated using a standard Fourier inversion. In the case with a mean flow the energy inversion is solved using a MINRES iteration [41] applied to a preconditioned form of the mean eddy energy equation

$$
[-\nabla \cdot(\mathbf{K} \nabla \cdot)+2]^{-1} \overline{\boldsymbol{u}} \cdot \nabla \bar{e}_{\theta, \kappa}+\bar{e}_{\theta, \kappa}=[-\nabla \cdot(\mathbf{K} \nabla \cdot)+2]^{-1}\left(\bar{F}_{E}-2 \nu \bar{z}+\overline{\boldsymbol{u}} \cdot\left[\nabla \cdot\left(\overline{\boldsymbol{u}^{\prime} \otimes \boldsymbol{u}^{\prime}}\right)+\frac{\nabla \bar{p}_{u^{\prime}}}{\rho_{0}}\right]\right)
$$

The residual of this equation has units of energy, and the iteration is applied until the residual norm is less than $10^{-6}$. 

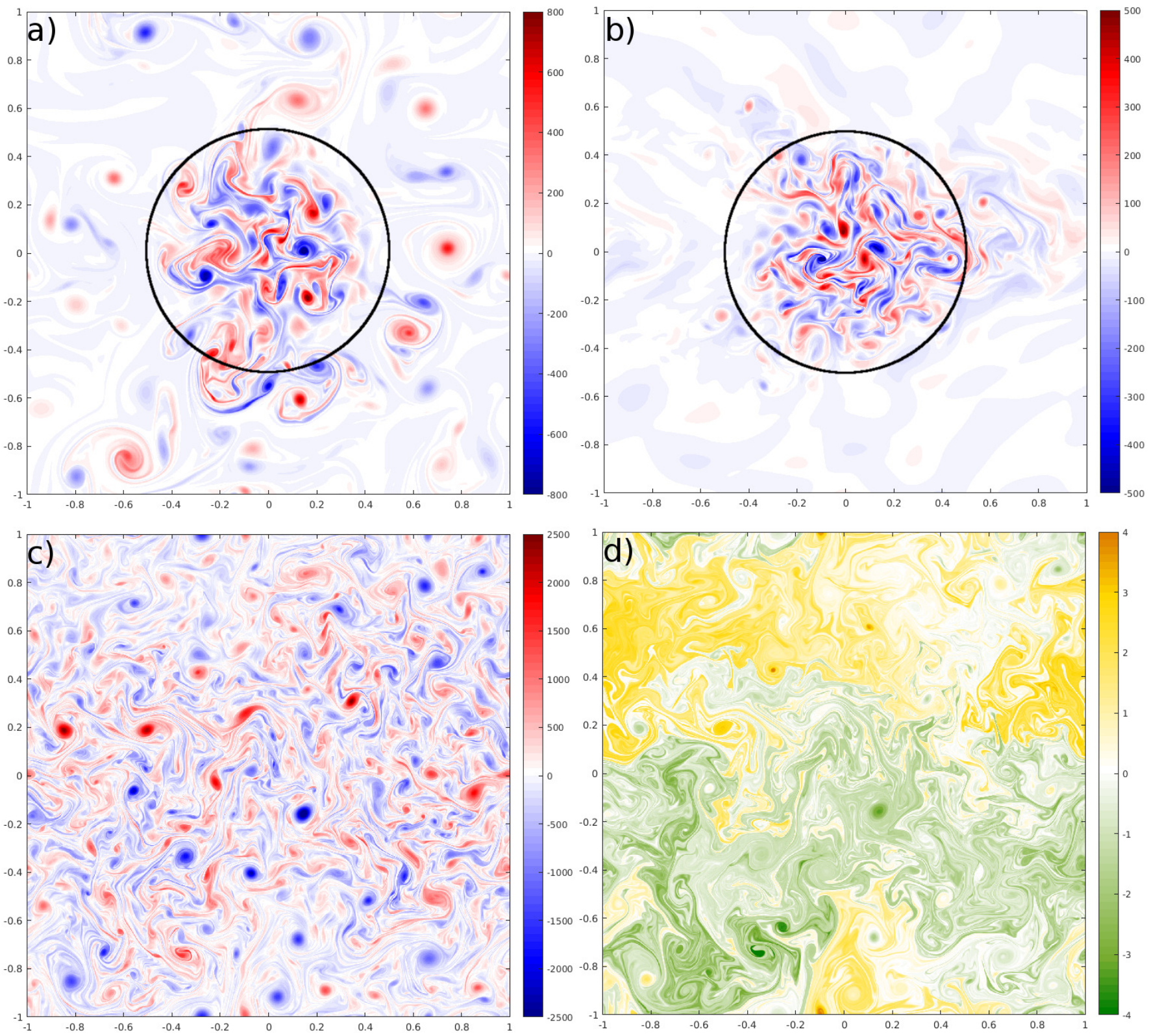

FIG. 1. Snapshots of vorticity $q$ for (a) $\lambda_{f}=1 / 8, F_{0}=10, \beta=0$ (b) $\lambda_{f}=1 / 10, F_{0}=5, \beta=500$ (c) $\lambda_{f}=1 / 10, F_{0}=15.66$, $\beta=0$. (d) shows a snapshot of tracer $C$ for the same simulation as (c). The circles in (a) and (b) are the boundaries of the forcing regions, outside of which there is no forcing.

\section{RESULTS}

Figure 1 shows snapshots of vorticity $q$ for three representative simulations: (a) shows an $f$-plane simulation with the circularly tapered forcing, (b) shows a $\beta$-plane simulation with circularly tapered forcing, and (c) shows an $f$-plane simulation with $x$-homogeneous forcing. Panel (d) shows the tracer $C$ from the same simulation shown in panel (c). The circles in panels (a) and (b) show the boundaries of the forcing region: outside the circles there is no forcing. The following three subsections present the results of the energetic analysis of the three configurations: first $f$-plane with circular forcing and no tracer, then $f$-plane with $x$-homogeneous forcing and a tracer, and finally $\beta$-plane with circular forcing. 


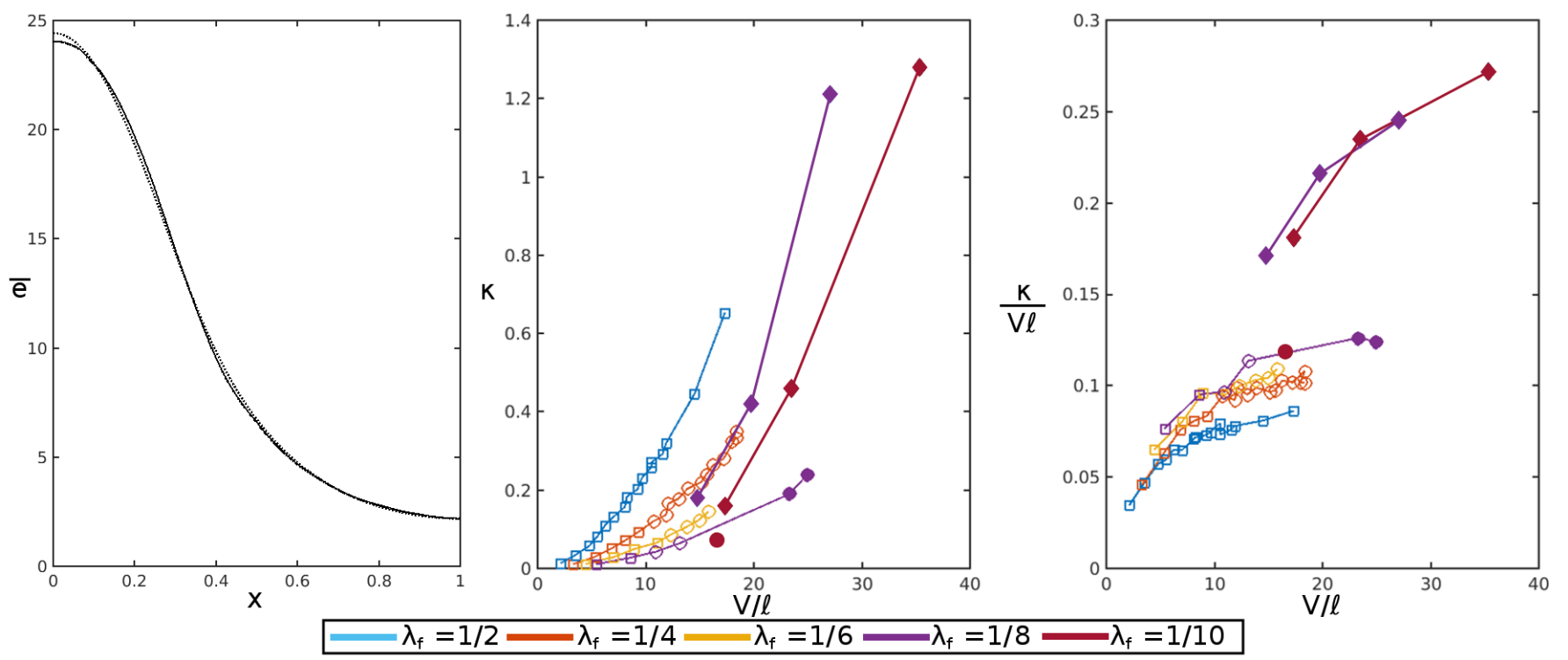

FIG. 2. The left panel shows the time-mean energy distribution $\bar{e}=\overline{\left|\boldsymbol{u}^{\prime}\right|^{2}} / 2$ along the line $y=0, x \in[0,1]$ as diagnosed from the simulation with $\lambda_{f}=1 / 8$ and $F_{0}=10$ (solid) and as implied by the optimized transport model (dotted). The center panel shows the diagnosed eddy energy diffusivity $\kappa$ as a function of the 'degree of nonlinearity' $V / \ell$, and the right panel shows the compensated diffusivity $\kappa /(V \ell)$. Colors denote the length scale of the forcing $\lambda_{f}$, as shown in the legend, while the marker shape encodes details of the geometry. Squares are simulations with domain width $L=3$ and circular forcing regions. Circles are simulations with $L=2$ and circular forcing. Diamonds are simulations with $L=2$ and $x$-homogeneous forcing. Filled-in markers denote new simulations not described previously in [39].

\section{A. Isotropic energy diffusion on the $f$-plane}

The $f$-plane simulations do not develop time-mean flows, and display isotropic, harmonic diffusion of eddy energy [39]. The energy transport model takes the simpler form $\mathbf{K}=\kappa \mathbf{I}$, with only one parameter to fit, the energy diffusivity $\kappa$. The time-mean energy distribution implied by the optimal $\kappa$ is typically extremely accurate; the left panel of Fig. 2 compares the true $\bar{e}$ (solid) and the optimal-model $\bar{e}$ (dotted) along the line $y=0, x \in[0,1]$ for the simulation with $\lambda_{f}=1 / 8$ and $F_{0}=10$. The lines are nearly indistinguishable, with a small discrepancy near $x=0$. One might argue that since the diffusivity $\kappa$ is related to the energy of the flow, which is highly inhomogeneous, the diffusivity should not be constant, but rather spatially-varying. The accuracy of the constant-diffusivity approximation evidenced in the left panel of Fig. 2 implies that there is very little to be gained by making $\kappa$ spatially-varying. This point is

There are always multiple ways of defining empirical 'characteristic' length and velocity scales, even in homogeneous turbulence. Here the turbulence is inhomogeneous, and is not described by a single length scale or amplitude. Nevertheless, an eddy velocity $V$ is here defined as the maximum value of $\sqrt{2 \bar{e}}$ (whereas in [39] $V$ was defined with respect to a spatially-averaged energy). The eddy length scale is defined as an integral scale of kinetic energy

$$
\ell=2 \pi \frac{\int E(k) \mathrm{d} k}{\int k E(k) \mathrm{d} k}
$$

where $E(k)$ is the one-dimensional energy spectrum. These amplitude and length estimates permit an estimate of the degree of nonlinearity of the turbulence: the advective time scale is roughly $\ell / V$, while the frictional time scale (which dominates the damping, viscosity being weak) is nondimensionalized to 1 . When their ratio $V / \ell$ is large the dynamics are strongly nonlinear; in [39] this ratio was called the 'frictional Reynolds number,' and was computed using a different definition of $V$.

The center panel of Fig. 2 plots the optimal $\kappa$ as a function of the degree of nonlinearity $V / \ell$ for all the simulations in [39] as well as the new $f$-plane simulations. Solid symbols denote new simulations while hollow symbols denote simulations described in [39]. Square symbols denote simulations in a square domain of length $L=3$, circles denote simulations in a square domain of length $L=2$, and diamonds denote simulations in a square domain of length $L=2$ with $x$-homogeneous forcing. The range of diagnosed energy diffusivities is large, spanning more than an order of 

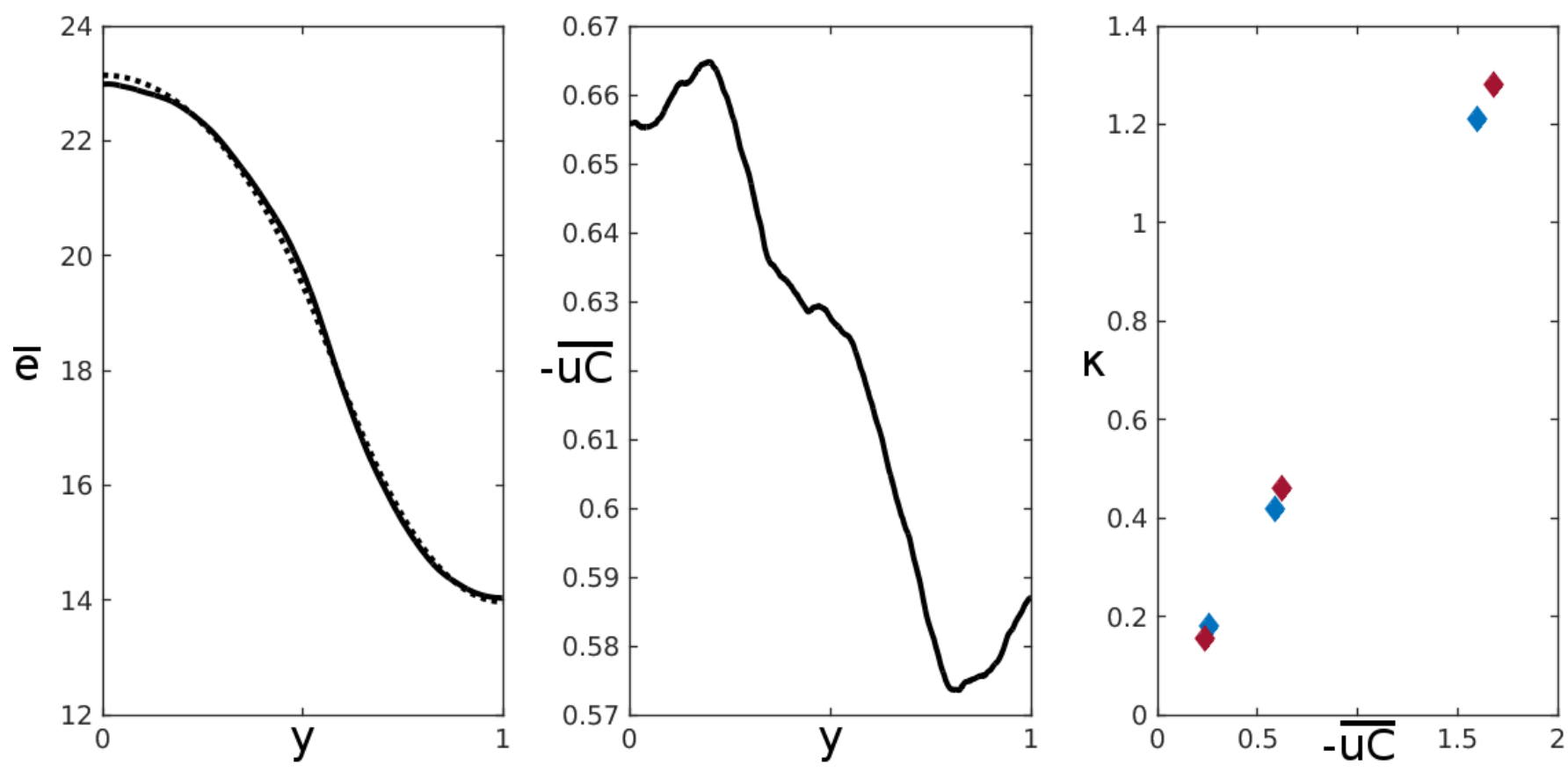

FIG. 3. Left: Diagnosed mean eddy energy profile (solid) and diffusive model fit (dotted) from the simulation with $x$ homogeneous forcing, $\lambda_{f}=1 / 10$, and $F_{0}=7.84$. Center: Time-mean of $-\overline{u C}$ for the same simulation. Right: Scatter plot of energy diffusivity versus mean tracer diffusivity for the six tracer simulations.

magnitude, and all of the new simulations are strongly nonlinear.

The right panel of Fig. 2 plots the compensated diffusivity $\kappa /(V \ell)$ as a function of the degree of nonlinearity $V / \ell$. If the diagnosed diffusivities were well described by a mixing length approximation $\kappa \propto V \ell$ the data points would all lie along a horizontal line. While this is clearly not the case, the mixing-length scaling does a reasonably-accurate job of collapsing the very-wide range of diffusivities shown in the center panel of Fig. 2. When the degree of nonlinearity is sufficiently strong $(V / \ell \geq 10)$ the diffusivities of simulations with circular forcing are all modeled by $\kappa=\gamma V \ell$ with $\gamma=0.1 \pm 0.03$ (in [39] the proportionality coefficient $\gamma$ was called $C$; here $C$ denotes a tracer concentration). Within any single experimental configuration (e.g. $\lambda_{f}=1 / 8$ with a circular forcing) the proportionality coefficient $\gamma$ seems to approach a limiting value with increasing energy. Different configurations have different limiting values, e.g. the limiting $\gamma$ for $\lambda_{f}=1 / 6$ appears to be different from that for $\lambda_{f}=1 / 10$. But the differences in $\gamma$ tend to decrease as the forcing scale decreases (for simulations with circular forcing): $\gamma$ for $\lambda_{f}=1 / 10$ is far more similar to $\lambda_{f}=1 / 8$ than to $\lambda_{f}=1 / 2$. This suggests that finite-size effects may be at play: the mixing-length model is more universal when there is a scale separation between the scale of the eddies and the scale of the flow inhomogeneity (i.e. the scale of the forcing region).

The proportionality coefficient in simulations with circular forcing is significantly smaller than the coefficient in simulations with $x$-homogeneous forcing. It may be the case that there is a different definition of the 'characteristic' velocity and length scales $V$ and $\ell$ that correctly describes the velocity and length scales that are important for energy diffusion. If this were the case, then it might further imply that the 'correct' proportionality coefficient $\gamma$ would be similar across all simulations. The definitions of $V$ and $\ell$ chosen here give a self-consistent view of velocity and length scales when applied to a single kind of inhomogeneous flow, but not when comparing flows with different kinds of inhomogeneity. Put simply, two flows with the same maximum velocity $V$ can be very, very different in other important ways. The goal here is not to ascertain optimal definitions for $V$ and $\ell$, but more simply to argue that a mixing-length scaling $\kappa \propto V \ell$ is a reasonable description of the data.

\section{B. Tracer versus energy diffusion}

Passive tracer transport in homogeneous turbulence is well-known to act diffusively over long-enough time scales 
fact that energy has been observed to be transported diffusively is suggestive. The tracer experiments described in section II A were designed to permit a simultaneous diagnosis of energy and tracer diffusivity. The tracer evolves in the presence of a constant, unit-amplitude $x$-directed tracer gradient. The vorticity forcing is homogeneous in the $x$ direction, so that the long-time statistics of the dynamics are $x$-invariant. The dynamics generate a mean tracer flux $\overline{u C}$ that is independent of $x$. The tracer diffusivity implied by the mean tracer flux is equal to minus the flux divided by the gradient, i.e. $\kappa_{C}=-\overline{u C}$, since the gradient has unit amplitude. Since the dynamics are on an $f$-plane, and the previous experiments indicate that isotropic diffusion $\mathbf{K}=\kappa \mathbf{I}$ is an accurate model, the energy diffusivity $\kappa$ is diagnosed the same way as in the previous section, and the values of the diagnosed diffusivity are shown in Fig. 2.

Figure 3 shows results for the tracer simulations. The left panel shows the true time-mean energy distribution (solid) and the prediction using a fitted Laplacian diffusion (dotted) as functions of $y \in[0,1]$ (the left half of the interval is symmetric) for the simulation with $\lambda_{f}=1 / 10$ and $F_{0}=7.84$. As in the case with circular forcing, the agreement is excellent, showing that Laplacian diffusion with a constant coefficient is an accurate model. The center panel shows the time-mean tracer diffusivity $-\overline{u C}$, also as a function of $y \in[0,1]$ for the same simulation. The tracer distribution. The distribution is also not very smooth, which suggests that a longer time average might be necessary. This is despite the fact that the time average has been computed over 534 time units; the eddy turnover time is $\ell / V \approx 1 / 25$, so the average has already been taken over more than 13,000 eddy turnover times. All six simulations have results similar to those in the left and center panels of Fig. 3: the diffusive model for energy transport is very accurate, and tracer diffusivity is spatially-variable.

The right panel of Fig. 3 plots eddy energy diffusivity as a function of tracer diffusivity for the six simulations. Since the value of tracer diffusivity varies over the domain, the spatially-averaged value is used for the plots. The two diffusivities are clearly related, and the best-fit linear relationship has the form $\kappa=0.75(-\overline{u C})$.

\section{Energy transport on the $\beta$-plane}

The addition of a constant background vorticity gradient $\beta$ brings significant changes to the dynamics. On the $f$-plane, the primary means of energy propagation outside of the forcing region is the ballistic motion of pairs of dipole vortices. On the $\beta$-plane low-energy Rossby waves transport energy at the group velocity, monopole vortices propagate by radiating Rossby waves, and dipole pairs have a preferred direction of propagation [30]. In addition, the eddy turbulence becomes anisotropic and forces a mean flow that in turn advects and distorts the eddy turbulence.

Figure 4 shows the results of the $\beta$-plane simulation with $\lambda_{f}=1 / 10, F_{0}=5$, and $\beta=500$. The upper right panel shows the eddy energy generation term $\overline{\mathcal{G}}$ (defined in Eq. 12). This term is negative over almost the entire domain, indicating that the eddies lose energy to the mean flow almost everywhere. The rate of energy loss is greatest just to the right of the forcing region. The upper left panel shows the time-mean streamfunction $\bar{\psi}$ generated by this eddy forcing. The mean flow consists of a fairly narrow positive (rightward) zonal jet through the center of the domain, with weak return (leftward) flow on the flanks. The time-mean flow is associated with a time-mean vorticity gradient that reaches a maximum of 318 , comparable to $\beta$. To assess the relative importance of nonlinear eddy-eddy interaction and linear wave dynamics, the following ratio was computed

$$
\frac{\left\|u^{\prime} \partial_{x} \bar{q}+v^{\prime} \partial_{y} \bar{q}+\beta v^{\prime}\right\|_{2}}{\left\|u^{\prime} \cdot \nabla q^{\prime}\right\|_{2}}
$$

where the $L^{2}$ norm was computed on the square $(x, y) \in[-1 / 2,1 / 2] \times[-1 / 2,1 / 2]$. For this simulation the value is 0.04 , indicating that nonlinear eddy-eddy advection dominates linear wave dynamics in the center of the domain. This ratio increases with $\beta$, but at $\beta=3000$ it is still only 0.19 .

The lower left panel of Fig. 4 shows the time-mean eddy energy $\bar{e}$. It clearly shows the effects of mean-flow advection, with the central peak carried rightward in the center of the domain and a slight leftward tendency near the upper and lower edges. The advective-diffusive transport model with an anisotropic diffusivity was optimized with a diagonal diffusion matrix $\mathbf{K}$ (i.e. $\theta=0$ in Eq. 15) with $\kappa_{1}=0.07$ in the $x$ direction and $\kappa_{2}=0.04$ in the $y$ direction. The main ficiencies of the advective-diffusive transport model are that the energy is diffused a bit too much in the center, while the far-field energy predicted by the model is too small, but the advective-diffusive model remains reasonably accurate.

The optimal diffusion matrices obtained for the four simulations at $\lambda_{f}=1 / 10$ are all diagonal, with (i) $\kappa_{1}=0.09$, $\kappa_{2}=0.05$ at $\beta=1000$, (ii) $\kappa_{1}=0.11, \kappa_{2}=0.08$ at $\beta=2000$, and (iii) $\kappa_{1}=0.14, \kappa_{2}=0.09$ at $\beta=3000$. The results

360 at higher $\beta$ are qualitatively similar to those shown in Fig. 4, but the quality of the approximated energy distribution degrades somewhat at higher $\beta$.

Anisotropy of the diffusion is not surprising given the ways that nonzero $\beta$ alters the dynamics. Indeed one might suspect that linear dynamics alone might give an anisotropic energy distribution; a brief digression is made here 

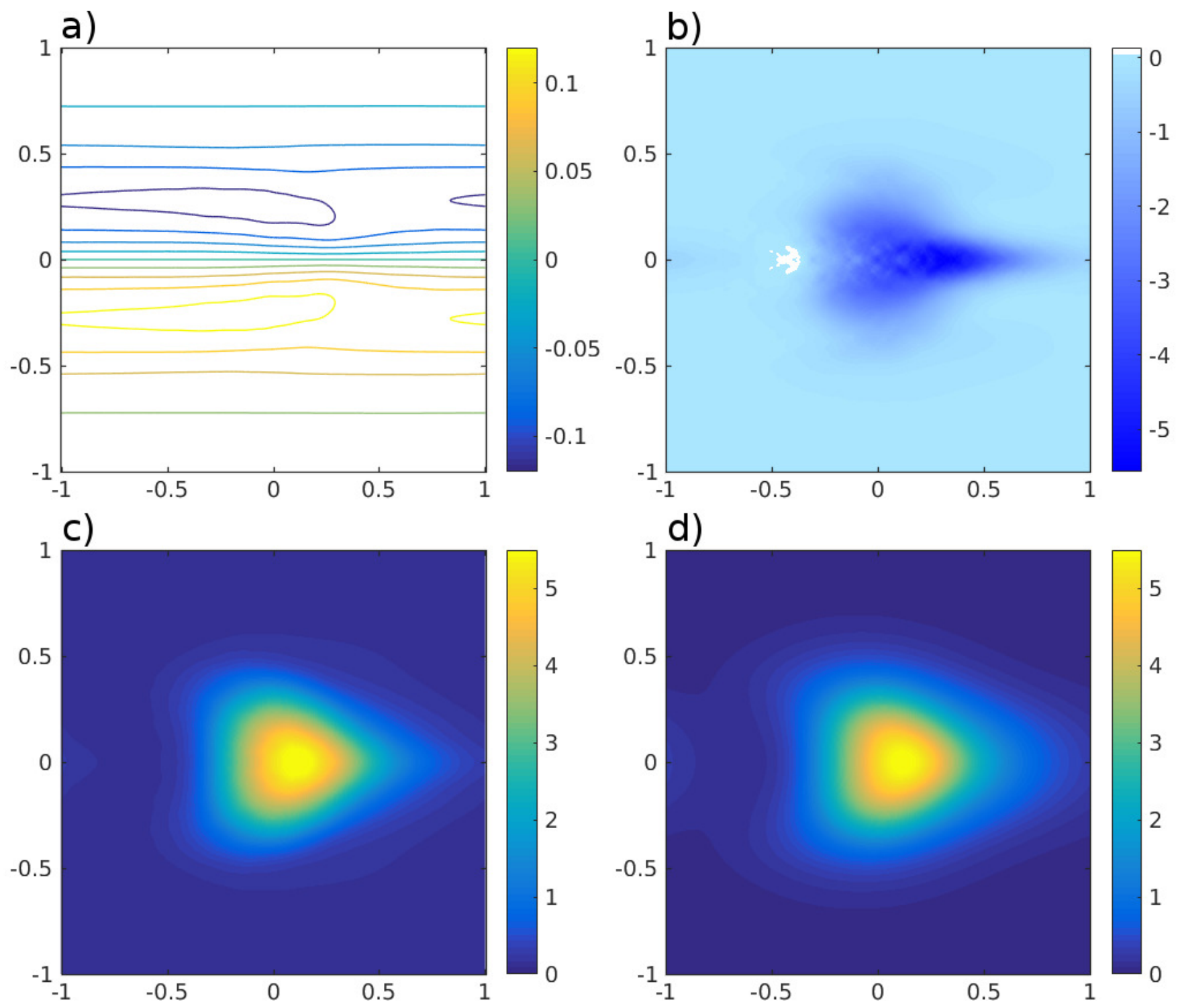

FIG. 4. Results for the simulation with $\lambda_{f}=1 / 10, F_{0}=5$, and $\beta=500$. a) Contours of time-mean streamfunction $\bar{\psi}$; the countour interval is 0.03 and the minimum contour is -0.12 . b) The eddy 'generation' term $\overline{\mathcal{G}}$; negative values indicate that eddies transfer energy to the mean flow. c) The time-mean eddy energy $\bar{e}$. d) The time-mean eddy energy from an optimized advective-diffusive transport model with $\kappa_{1}=0.07, \kappa_{2}=0.04, \theta=0$ (see Eq. 15). The color scale is the same in c) and d).

to show that this is not the case. An infinitesimal-amplitude plane wave of the form $\psi(\boldsymbol{x}, t)=\hat{\psi} e^{\mathrm{i}(\boldsymbol{k} \cdot \boldsymbol{x}-\omega t)}$ evolves according to the barotropic, rigid-lid dispersion relation

$$
\omega=-\beta \frac{k_{x}}{k_{x}^{2}+k_{y}^{2}}
$$

The linear dynamics of a wave packet transport energy with the group velocity [45], which is

$$
\boldsymbol{c}_{g}=\frac{\beta}{k^{2}}\left(\begin{array}{c}
\cos (2 \phi) \\
\sin (2 \phi)
\end{array}\right)
$$

where $k_{x}=k \cos (\phi)$ and $k_{y}=k \sin (\phi)$. The group velocity thus clearly depends on the orientation of the wave, but the magnitude of the group velocity is simply $\beta / k^{2}$, which is independent of the orientation of the wave. A stochastic source of wave packets with uniform distribution over $\phi$ would thus produce an isotropic mean energy distribution; 

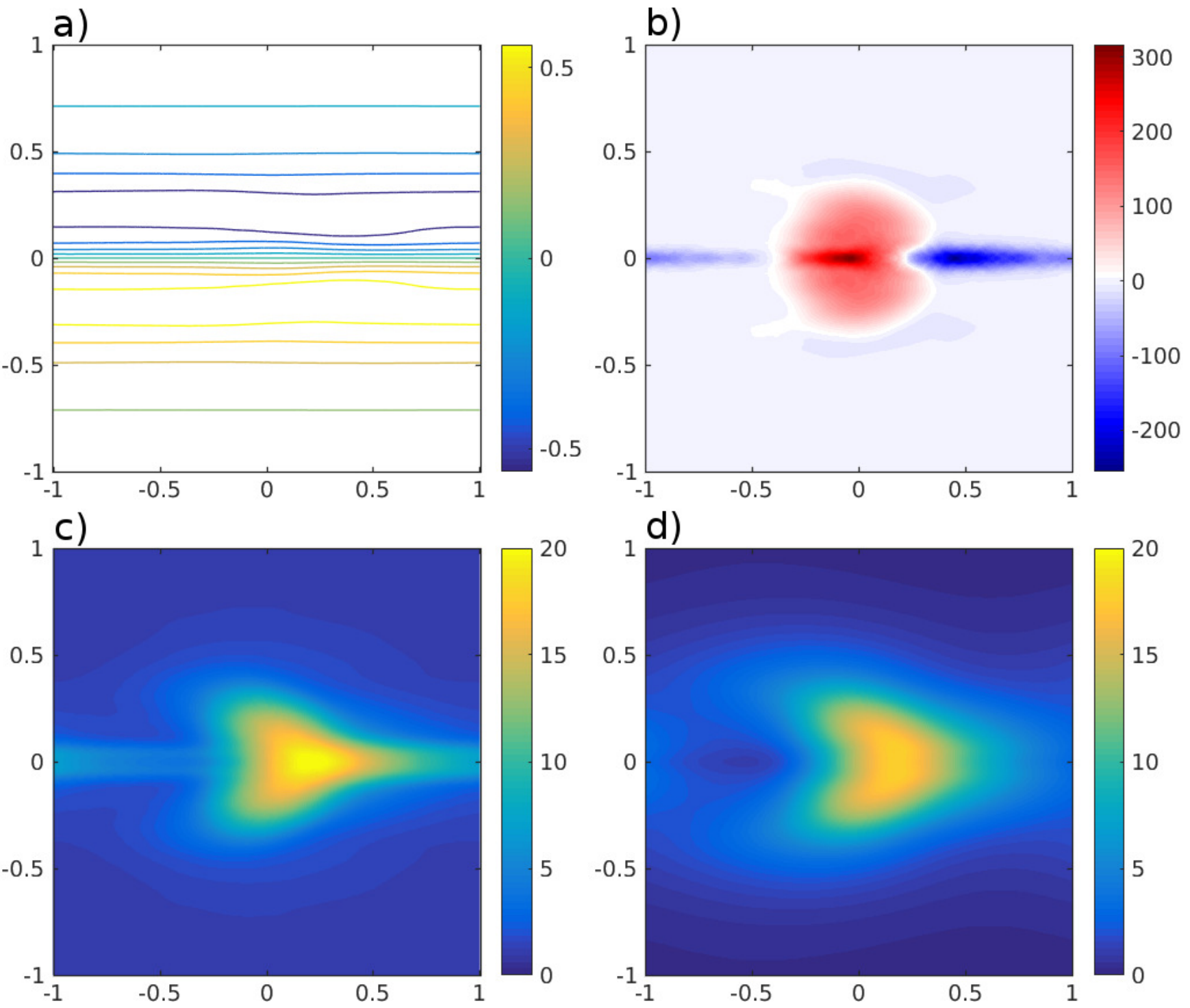

FIG. 5. Results for the simulation with $\lambda_{f}=1 / 8, F_{0}=10$, and $\beta=2000$. a) Contours of time-mean streamfunction $\bar{\psi}$; the countour interval is 0.14 and the minimum contour is -0.56 . b) The sum of energy forcing terms $\bar{F}_{E}-2 \nu \bar{z}+\overline{\mathcal{G}}$. c) The time-mean eddy energy $\bar{e}$. d) The time-mean eddy energy from an advective-diffusive transport model with $\kappa_{1}=0.2, \kappa_{2}=0.1$, $\theta=0$ (see Eq. 15). The color scale is the same in c) and d).

this has been verified here by running a simulation of the stochastically-forced linear dynamics (results not shown).

The snapshot of vorticity in the upper right panel of Fig. 1 suggests that linear Rossby wave dynamics are operating in the far-field region, which may help explain the higher-than-diffusive transport of energy to the far field. But the foregoing argument shows that linear wave dynamics are not by themselves responsible for the anisotropy observed here. Allowing a free surface or baroclinicity would alter the dispersion relation to $\omega=-\beta k_{x} /\left(k_{d}^{2}+k_{x}^{2}+k_{y}^{2}\right)$ for some deformation wavenumber $k_{d}$, and the linear dynamics themselves would generate an anisotropic energy distribution.

Figure 5 shows the results of the simulation at $\lambda_{f}=1 / 8, F_{0}=10$, and $\beta=2000$. The panels are the same as Fig. 4 except that the upper right panel now shows the sum of the energy forcing, viscous dissipation, and mean-flow loss terms $\bar{F}_{E}-2 \nu \bar{z}+\overline{\mathcal{G}}$. The energy is significantly higher, as is $\beta$. The ratio of the linear eddy interaction with the mean vorticity gradient to the nonlinear eddy-eddy interaction (expression 19) is only 0.065 , indicating strongly nonlinear flow in the forcing region. The time-mean flow consists of a thin, fast zonal jet in the center of the domain, with return flow on the flanks, and the eddy energy distribution is stretched rightward in the center and leftward at 
the top and bottom. The mean jet is not much wider than an eddy scale; the forcing wavelength is $\lambda_{f}=1 / 8$ and varies slightly over the domain, but remains quite thin.) The model energy distribution shown in the lower right panel was generated with a diagonal diffusion matrix with $\kappa_{1}=0.2$ and $\kappa_{2}=0.1$, and represents an imperfect 'optimized' model. The model energy distribution in the lower right panel is a poor approximation to the true energy distribution both in the core near the center, which is too weak and has the wrong shape, and in the far field, which has too little tend to reduce the $L^{1}$ and $L^{2}$ norm of the error by increasing the energy in the far field, while at the same time increasing the $L^{\infty}$ norm by diffusing away too much of the central energy peak. The same behavior is observed in the simulation at $\lambda_{f}=1 / 8, F_{0}=10$, and $\beta=1000$. Possible reasons for the failure of the advection-diffusion model of energy transport in these simulations are discussed in the next section.

This research continues the experimental investigation of eddy energy transport begun in [39]. Ultimately the goal is to improve parameterizations of mesoscale eddy dynamics in coarse-resolution ocean general circulation models. These models typically parameterize the effects of mesoscale eddy tracer transport within the context of the combined GentMcWilliams [6, GM] and Redi [8] frameworks. The GM framework in particular includes a quasi-Stokes diffusivity coefficient $\kappa_{\mathrm{GM}}$, and recent investigations have argued that the spatial and temporal variation of $\kappa_{\mathrm{GM}}$ should be related to that of the eddy energy [15-23]. There is evidence that horizontal transport plays a non-negligible role in setting the global distribution of eddy energy [26-28], which implies the need for a prognostic eddy energy model. Eden \& Greatbatch [16] and Marshall \& Adcroft [17] have proposed one-equation models for mesoscale eddy energy. In both of these models the eddy energy is transported by a combination of mean-flow advection and isotropic diffusion, which is a common model of eddy energy transport in modeling three-dimensional, non-geostrophic turbulence [24, 35-38]. Geostrophic turbulence at the ocean mesoscales is qualitatively different from the isotropic homogeneous turbulence at smaller scales where subgrid-scale turbulent kinetic energy transport has previously been modeled as advection plus diffusion, so it is not obvious that mesoscale eddy energy should be transported in the same way.

The author began an investigation into the transport of mesoscale eddy energy in [39], which has been continued here. The investigations have thus far been carried out in the context of barotropic, rigid-lid dynamics, which is a minimal model qualitatively capturing certain features of the ocean mesoscale. The approach taken in these investigations is to use stochastic forcing to generate an inhomogeneous turbulence field. The time-mean eddy energy distribution is diagnosed, and energy transport models are fit to the data by minimizing the error between the model's prediction and the observed distribution of time-mean eddy energy. The initial investigation [39] discovered that isotropic diffusive transport is an accurate model in the absence of a mean flow and in the absence of a background vorticity gradient (i.e. $f$-plane dynamics, $\beta=0$ ). Furthermore, the energy diffusivity $\kappa$ was reasonably well modeled using a mixing-length model $\kappa \propto V \ell$ where $V$ and $\ell$ are characteristic velocity and length scales of the turbulence.

The success of a mixing-length model for energy transport suggests that energy might be transported like a passive tracer, since the mixing-length diffusion model is a model of tracer transport ${ }^{1}$ [42, 43]. This question was addressed here by adding a passive tracer to the barotropic dynamics. Surprisingly, the diagnosed energy diffusivity was invariably smaller than the tracer diffusivity; the energy diffusivity was close to $75 \%$ of the tracer diffusivity in all simulations. This relationship between tracer diffusivity and energy diffusivity strongly suggests that one might construct a theory that both justifies a diffusive model for energy transport and relates it to existing theories for tracer transport. No such theory is advanced here, leaving the question open.

As noted above, $f$-plane barotropic dynamics is a minimal model of ocean mesoscale eddy dynamics. It lacks two crucial features of mesoscale dynamics: vertical density stratification and a large-scale potential vorticity gradient. The effect of vertical stratification has not been investigated, but has the potential to lead to fundamental changes ecause of the existence of propagating monopoles (hetons [46]) which are absent from barotropic flow on an $f$-plane.

The simplest way to extend the barotropic model is to move from the $f$-plane to the $\beta$-plane. An immediate consequence of this change is that the system generates a time-mean flow, introducing mean-flow interactions that were avoided on the $f$-plane. This mean-flow generation is itself a consequence of anisotropization of the eddy dynamics, since an isotropic eddy field does not generate a mean flow. Additionally, the addition of $\beta$ causes monopole vortices to propagate westward and Rossby waves to transport energy. It is perhaps natural to assume that the eddy energy

\footnotetext{
1 The question of whether the energy and tracer diffusivities are related was raised by an anonymous reviewer of [39].
} 
transport might continue to be well-modeled by mean-flow advection plus anisotropic diffusion, where the diffusion coefficient $\kappa$ is replaced by a $2 \times 2$ symmetric positive definite matrix. The results, however, are mixed. At low to moderate levels of eddy energy the core of the eddy energy distribution (i.e. the high-energy region near the peak of the forcing) is reasonably well reproduced by an energy transport model consisting of mean-flow advection plus anisotropic diffusion, but the low-energy regions far from the forcing are poorly predicted by a diffusive model. At higher levels of eddy energy the advection-diffusion model predicts the eddy energy distribution quite poorly, both in the high-energy core and in the low-energy far field.

There are at least three possible reasons for the failure of the advection-diffusion model on the $\beta$-plane. In the first place, the energy diffusivity used here is constant despite the fact that the turbulence is highly inhomogeneous, the diffusivity was found (at least on the $f$-plane) to be proportional to an eddy velocity scale. There are two reasons to suspect that this may not be the reason for the observed failure on the $\beta$-plane. On one hand, a constant diffusivity is remarkably accurate on the $f$-plane, despite the significant level of inhomogeneity. On the other hand, a transport model whose energy flux decreases with the energy (as does the mixing-length model) would be worse than the constant-coefficient diffusivity model. The constant-coefficient model predicts a too-weak core and a too-weak far field. The variable-coefficient model would either predict even less flux to the far field or even more flux away from the core, and therefore could not improve on the constant-coefficient model.

A second possible reason for the failure of the advection-diffusion model on the $\beta$-plane is that the diffusive transport model does not describe transport by linear (or weakly-nonlinear) waves. Diffusive transport is conceptually associated with random-walk movement in a velocity field generated by strongly nonlinear, turbulent dynamics. Rossby-wave dynamics describe the low energy regions of the $\beta$-plane model, and the transport of energy by linear wave dynamics is ballistic rather than diffusive. This deficiency may explain why the diffusive model predicts too little energy in the far field, away from the forcing region. It might be possible to construct a transport model hybridizing diffusive transport in high-energy regions and wavelike transport in low-energy regions (cf. the wave-energy transport model in $[47])$.

A third possible reason for the failure of the advection-diffusion model on the $\beta$-plane is the breakdown of scale separation between the mean flow and eddies observed in the higher-energy experiments reported in section III C. As noted above, diffusive behavior is conceptually related to chaotic motion in a turbulent velocity field. In geostrophic turbulence this chaotic motion is associated with a large population of interacting eddies. In some of the simulations reported here, the eddy energy is elevated in a very thin region, within the core of a thin mean jet that is sweeping eddies out of the forcing region. This region is not wide enough to contain a large population of interacting vortices, and the eddy energy transport, rather than being diffusive, depends crucially on the dynamics of a single vortex (or a small number of vortices) within a highly inhomogeneous environment.

From another perspective, the diffusive transport models fail even on the $f$-plane, where they are able to accurately predict the time-mean eddy energy profile. This is because the mean eddy distribution bears little resemblance to the actual eddy field at any point in time; the mean is not a particularly useful summary statistic for eddy energy. For example, in the $f$-plane simulations with circular forcing the low energy regions are only occasionally visited by vortex dipole pairs. The low value of time-mean energy in those regions thus results from the occasional passage of eddies with energies well above the mean level. This discrepancy between the time-mean energy and the energy of a typical eddy may also help explain why a constant diffusivity $\kappa$ is so successful in a simulation whose mean energy field is highly inhomogeneous: because the energy of a typical eddy does not vary much over the domain.

Further evidence of the deficiency of the time-mean energy as a useful descriptor of the dynamics is provided by the length of time it takes to compute the time-mean eddy energy. In a moderately-energetic simulation the eddy turnover time is $\ell / V \sim 1 / 20$ and it takes at least 300 units of time averaging (started after the total energy has equilibrated) is presumably related to the fundamental differences between homogeneous, isotropic, three-dimensional turbulence and geophysical turbulence with long-lived coherent vortices, which also leads to anomalous long-tail behavior in tracer transport [44, 48]. In light of this more fundamental deficiency, and in light of the goal of developing a model to predict the actual eddy energy distribution (rather than the mean eddy energy distribution), future work will

focus on stochastic modeling of the eddy energy transport. Such an effort, if successful, may as a byproduct lead to an improved model of the mean transport on the $\beta$-plane, and would naturally fit into the ongoing development of stochastic parameterizations for mesoscale eddy effects [49]. 
[1] D. B. Chelton, M. G. Schlax, and R. M. Samelson, "Global observations of nonlinear mesoscale eddies," Prog. Oceanogr. 91, 167 (2011).

[2] S. M. Griffies, M. Winton, W. G. Anderson, R. Benson, T. L. Delworth, C. O. Dufour, J. P. Dunne, P. Goddard, A. K. Morrison, A. Rosati, A. Wittenberg, J. Yin, and R. Zhang, "Impacts on ocean heat from transient mesoscale eddies in a hierarchy of climate models," J. Climate 28, 952 (2015).

[3] J. Kay, C. Deser, A. Phillips, A. Mai, C. Hannay, G. Strand, J. Arblaster, S. Bates, G. Danabasoglu, J. Edwards, M. Holland, P. Kushner, J.-F. Lamarque, D. Lawrence, K. Lindsay, A. Middleton, E. Munoz, R. Neale, K. Oleson, L. Polvani, and M. Vertenstein, "The Community Earth System Model (CESM) large ensemble project: A community resource for studying climate change in the presence of internal climate variability," Bull. American Meteorol. Soc. 96, $1333(2015)$.

[4] S. Griffies, A. Biastoch, C. Böning, F. Bryan, G. Danabasoglu, E. Chassignet, M. England, R. Gerdes, H. Haak, R. Hallberg, W. Hazeleger, J. Jungclaus, W. Large, G. Madec, A. Pirani, B. Samuels, M. Scheinert, A. Sen Gupta, C. Severijns, H. Simmons, A. Treguier, M. Winton, S. Yeager, and J. Yin, "Coordinated ocean-ice reference experiments (COREs)," Ocean Model. 26, 1 (2009).

[5] A. R. Karspeck, S. Yeager, G. Danabasoglu, T. Hoar, N. Collins, K. Raeder, J. Anderson, and J. Tribbia, "An ensemble adjustment Kalman filter for the CCSM4 ocean component," J. Climate 26, 7392 (2013).

[6] P. R. Gent and J. C. McWilliams, "Isopycnal mixing in ocean circulation models," J. Phys. Ocean. 20, 150 (1990).

[7] P. R. Gent, J. Willebrand, T. J. McDougall, and J. C. McWilliams, "Parameterizing eddy-induced tracer transports in ocean circulation models," J. Phys. Ocean. 25, 463 (1995).

[8] M. H. Redi, "Oceanic isopycnal mixing by coordinate rotation," J. Phys. Ocean. 12, 1154 (1982).

[9] I. Held and V. Larichev, "A scaling theory for horizontally homogeneous, baroclinically unstable flow on a beta plane," J. Atmos. Sci. 53, 946 (1996).

[10] M. Visbeck, J. Marshall, T. Haine, and M. Spall, "Specification of eddy transfer coefficients in coarse-resolution ocean circulation models," J. Phys. Ocean. 27, 381 (1997).

[11] P. H. Stone, "A simplified radiative-dynamical model for the static stability of rotating atmospheres," J. Atmos. Sci. 29, 405 (1972).

[12] K. S. Smith, "The geography of linear baroclinic instability in earth's oceans," J. Marine Res. 65, 655 (2007).

[13] R. Tulloch, J. Marshall, C. Hill, and K. S. Smith, "Scales, growth rates, and spectral fluxes of baroclinic instability in the ocean," J. Phys. Ocean. 41, 1057 (2011).

[14] A. Klocker and R. Abernathey, "Global patterns of mesoscale eddy properties and diffusivities," J. Phys. Ocean. 44, 1030 (2014).

520 [15] P. Cessi, "An energy-constrained parameterization of eddy buoyancy flux," J. Phys. Ocean. 38 (2008).

[16] C. Eden and R. J. Greatbatch, "Towards a mesoscale eddy closure," Ocean Model. 20, 223 (2008).

[17] D. P. Marshall and A. J. Adcroft, "Parameterization of ocean eddies: Potential vorticity mixing, energetics and arnolds first stability theorem," Ocean Model. 32, 188 (2010).

[18] M. F. Jansen, A. J. Adcroft, R. Hallberg, and I. M. Held, "Parameterization of eddy fluxes based on a mesoscale energy budget," Ocean Model. 92, 28 (2015).

[19] D. P. Marshall, J. R. Maddison, and P. S. Berloff, "A framework for parameterizing eddy potential vorticity fluxes," J. Phys. Ocean. 42, 539 (2012).

[20] S. Bachman and B. Fox-Kemper, "Eddy parameterization challenge suite I: Eady spindown," Ocean Model. 64, 12 (2013).

[21] S. Bachman, D. Marshall, J. Maddison, and J. Mak, "Evaluation of a scalar eddy transport coefficient based on geometric constraints," Ocean Model. 109, 44 (2017).

[22] D. P. Marshall, M. H. Ambaum, J. R. Maddison, D. R. Munday, and L. Novak, "Eddy saturation and frictional control of the antarctic circumpolar current," Geo. Res. Lett. 44, 286 (2017).

[23] J. Mak, D. Marshall, J. Maddison, and S. D. Bachman, "Emergent eddy saturation from an energy constrained eddy parameterisation," Ocean Model. 112, 125 (2017).

[24] S. B. Pope, Turbulent flows (Cambridge university press, 2000).

[25] G. L. Mellor and T. Yamada, "Development of a turbulence closure model for geophysical fluid problems," Rev. Geophys. 20, 851 (1982).

[26] I. Grooms, L.-P. Nadeau, and K. S. Smith, "Mesoscale eddy energy locality in an idealized ocean model," J. Phys. Ocean. 43 (2013).

[27] R. Chen, G. R. Flierl, and C. Wunsch, "A description of local and nonlocal eddy-mean flow interaction in a global eddy-permitting state estimate," J. Phys. Ocean. 44, 2336 (2014).

[28] I. Grooms, K. S. Smith, and A. J. Majda, "Multiscale models for synoptic-mesoscale interactions in the oceans," Dyn. Atmos. Oceans 58 (2012), 10.1016/j.dynatmoce.2012.09.003.

[29] J. C. McWilliams and G. R. Flierl, "On the evolution of isolated, nonlinear vortices," J. Phys. Ocean. 9, 1155 (1979).

[30] G. Reznik, "Dynamics of localized vortices on the beta plane," Izvestiya, Atmospheric and Oceanic Physics 46, 784 (2010).

[31] X. Zhai, H. Johnson, and D. Marshall, "Significant sink of ocean-eddy energy near western boundaries," Nat. Geosci. 3, 
$608(2010)$.

[32] J. J. Early, R. Samelson, and D. B. Chelton, "The evolution and propagation of quasigeostrophic ocean eddies," J. Phys. Ocean. 41, 1535 (2011).

[40] G. Denk and S. Schäflne

Computing 59, 153 (1997).

[41] Y. Saad, Iterative methods for sparse linear systems (SIAM, 2003).

[43] A. Monin and A. Yaglom, Statistical Fluid Mechanics, Volume I: Mechanics of Turbulence (MIT Press, Cambridge MA, 1971).

[44] A. Provenzale, "Transport by coherent barotropic vortices," Annual Rev. Fluid Mech. 31, 55 (1999).

[45] O. Bühler, Waves and mean flows (Cambridge University Press, 2014).

[46] N. Hogg and H. Stommel, "The heton: an elementary interaction between discrete baroclinic geostrophic vortices, and its implications concerning eddy heat-flow," Proc. R. Soc. Lond. A 397, 1 (1985).

[47] D. Olbers and C. Eden, "A global model for the diapycnal diffusivity induced by internal gravity waves," J. Phys. Ocean. 43, 1759 (2013).

[48] J. Weiss, A. Provenzale, and J. McWilliams, "Lagrangian dynamics in high-dimensional point-vortex systems," Phys. Fluids 10, 1929 (1998).

[49] I. Grooms, "A Gaussian-product stochastic Gent-McWilliams parameterization," Ocean Model. 106, 27 (2016). 